\title{
Expert consensus: practical algorithms for management of inflammatory bowel disease patients presenting with back pain or peripheral arthropathies
}

\author{
Gaëlle Varkas $^{1,2}$ (D) | Clio Ribbens ${ }^{3}$ | Edouard Louis ${ }^{4}$ | Filip Van den Bosch ${ }^{1,2}$ | \\ Rik Lories $^{5}$ | Séverine Vermeire ${ }^{6}$ | Dirk Elewaut ${ }^{1,2}$ | Martine De $\operatorname{Vos}^{7}$ (D)
}

${ }^{1}$ Department of Rheumatology, Ghent University Hospital, Ghent, Belgium

${ }^{2}$ VIB Inflammation Research Center, Ghent University, Ghent, Belgium

${ }^{3}$ Department of Rheumatology, University of Liège, Liège, Belgium

${ }^{4}$ Department of Hepato-

Gastroenterology, CHU Liège, University of Liège, Liège, Belgium

${ }^{5}$ Laboratory of Tissue Homeostasis and Disease, Division of Rheumatology, Skeletal Biology and Engineering Research

Center, University Hospitals Leuven,

Leuven, Belgium

${ }^{6}$ Department of Clinical and Experimental

Medicine, Translational Research

Center for Gastrointestinal Disorders

(TARGID), University Hospitals Leuven,

Leuven, Belgium

${ }^{7}$ Department of Gastroenterology, Ghent

University Hospital, Ghent, Belgium

Correspondence

Prof. Dr. Martine De Vos, Department of Gastroenterology, Ghent University Hospital, De Pintelaan 185, Ghent 9000, Belgium.

Email: Martine.Devos@UZGent.be

Prof. Dr. Dirk Elewaut, Department of Rheumatology, Ghent University Hospital, De Pintelaan 185, Ghent 9000, Belgium. Email: dirk.elewaut@ugent.be

\section{Funding information}

This work was supported by AbbVie Belgium NV/SA. AbbVie funded all costs associated with the development and the publishing of this manuscript. The content was independently determined by the experts, without any company interference.

\section{Summary}

Background: Spondyloarthritis is the most frequent extra-intestinal manifestation of IBD.

Aim: To present simple strategies to identify and differentiate inflammatory joint pain in IBD patients.

Methods: A panel of Belgian gastroenterologists and rheumatologists developed seven algorithms for IBD patients with joint symptoms based on a Delphi exercise conducted between April and December 2016. Here, we focus on referral strategies for patients with chronic back pain (evidence-based strategy), large joint monoarthritis, oligo- or polyarticular arthritis or arthralgia (based on expert opinion). We also present management tools for IBD patients with acute back pain and small joint monoarthritis (Supplementary file).

Results: The reported algorithm for IBD patients with chronic back pain uses basic clinical criteria to identify which patients should be referred to the emergency room (spondylodiscitis), physical medicine and rehabilitation (mechanical back pain) or rheumatologist (spondyloarthritis). IBD patients with large joint monoarthritis should be referred to emergency room if septic arthritis is suspected; in other patients, blood analyses and referral to a rheumatologist for articular puncture with evacuation of synovial fluid are recommended. The analysis of synovial fluid allows for identification of non-inflammatory (e.g., osteoarthritis) and inflammatory (e.g., [pseudo]-gout, peripheral spondyloarthritis and Borrelia burgdorferi arthritis) conditions. In patients with inflammatory oligoarticular or polyarticular arthralgia, erythrocyte sedimentation rate, concomitant therapies, anti-nuclear factor and anti-double-stranded DNA antibody levels should be evaluated; in anti-tumour necrosis factor-treated patients, a drug-induced lupus-like syndrome should be considered.

Conclusion: We propose straightforward strategies for IBD patients with joint symptoms, which are specific enough to select initial treatment and referral pattern. 


\section{1 | INTRODUCTION}

IBD, including Crohn's disease and ulcerative colitis, affects not only the gut but also extra-intestinal sites in approximately one-third of patients, predominantly the skin and the joints. ${ }^{1,2}$ The most frequent extra-intestinal manifestation of IBD is spondyloarthritis, ${ }^{1}$ a group of articular chronic inflammatory diseases, including ankylosing spondylitis, psoriatic arthritis, reactive arthritis, IBD-related spondyloarthritis, and undifferentiated spondyloarthritis. ${ }^{3}$ IBD and spondyloarthritis are associated in genetic background, and in clinical and imaging features. ${ }^{4-6}$

Depending on the predominant manifestation, spondyloarthritis is divided into axial spondyloarthritis and peripheral spondyloarthritis. $^{7}$ The early diagnosis of spondyloarthritis may be a major challenge for physicians due to the lack of objective disease markers. ${ }^{8,9}$ Moreover, joint symptoms usually occur in patients treated with immunomodulatory drugs, masking clinical presentation. ${ }^{7}$ In axial spondyloarthritis, the main symptom is chronic (low) back pain induced by inflammation of the sacroiliac joints (sacroiliitis) or spine (spondylitis). In some patients, axial spondyloarthritis evolves into ankylosing spondylitis, a more severe disease stage with structural damage of the sacroiliac joints and/or spine visible on X-ray. ${ }^{10,11}$ The main symptoms of peripheral spondyloarthritis are arthritis, enthesitis and dactylitis. ${ }^{12}$

A recent meta-analysis has shown that up to $13 \%$ of IBD patients are diagnosed with peripheral arthritis, whereas prevalence estimates for sacroiliitis and ankylosing spondylitis are $10 \%$ and $3 \%$, respectively. ${ }^{12}$ Joint flares usually, but not exclusively, accompany gut flares. ${ }^{13,14}$ Although sacroiliitis can be seen on X-ray and computed tomography of the abdomen in IBD patients, ${ }^{15-17}$ recent studies have shown that magnetic resonance imaging is superior to detect inflammatory sacroiliitis and allows an earlier diagnosis of axial spondyloarthritis, before the occurrence of structural changes. ${ }^{18,19}$ However, the abnormalities of the sacroiliac joints present on magnetic resonance enterography were not associated with inflammatory back pain (IBP). ${ }^{19}$ Therefore, imaging findings should be interpreted cautiously in asymptomatic patients. Indeed, the significance of subclinical sacroiliitis is unknown and research has mainly focused on the diagnosis and treatment of symptomatic patients.

Considering the high prevalence of rheumatic diseases in IBD patients, the difficult diagnosis of spondyloarthritis and the shortage of rheumatologists, there is a need for feasible and simple referral strategies, sensitive and specific enough to select patients with rheumatic conditions suggestive of spondyloarthritis.

\section{2 | MATERIALS AND METHODS}

This paper has been developed based on a Delphi exercise amongst a panel of experts in the field of spondyloarthritis and/or IBD between April and December 2016.

First, the development of referral algorithms for IBD patients with joint symptoms was initiated by experts from the University of Ghent, who drafted initial referral proposals based on expert opinions, scientific literature and clinical experience. The relevant literature was selected through a thorough PubMed search, based on the following terms and their combinations: "inflammatory bowel disease", "arthritis", "sacroiliitis", "arthralgia", "referral", "spondyloarthritis" and "ankylosing spondylitis".

Secondly, a panel of eight interuniversity specialists in the field of IBD and spondyloarthritis, including Belgian gastroenterologists and rheumatologists from the Universities of Ghent, Leuven and Liège, were selected to attend a consensus meeting on May 2, 2016. Pairs of gastroenterologists and rheumatologists from the three universities discussed the proposed referral algorithms for common joint symptoms in IBD patients, such as acute back pain, chronic back pain, large joint monoarthritis, small joint monoarthritis, oligoor polyarthritis, monoarticular arthralgia and oligo- or polyarticular arthralgia. Based on the critical appraisal of the experts, the referral algorithms were revised. Updated proposals were shared with all experts who could provide additional feedback via email. Finally, the proposed referral algorithms were presented and tested for feasibility and applicability in a national meeting with nonselected gastroenterologists on December 2, 2016, leaving room for additional remarks from the audience.

Here, we focus on referral algorithms for IBD patients with chronic back pain, large joint monoarthritis and arthralgia, which are the most frequently encountered topics in clinical practice.

\section{3 | RESULTS AND DISCUSSION}

\section{1 | Axial spondyloarthritis}

Although axial spondyloarthritis is a common cause of chronic low back pain in IBD patients, its early diagnosis remains challenging. The proposed evidence-based referral algorithms for IBD patients with back pain are largely based on an existing algorithm developed for the overall population, but have been adapted to IBD patients taking into account their treatment. ${ }^{8}$

\subsection{1 | Exclusion of spondylodiscitis}

In IBD patients taking immunosuppressants, infectious spondylodiscitis must be excluded in case of new-onset back pain. In case of fever, high inflammatory parameters, infectious focus or neurological symptoms, immediate referral to the emergency room with a diagnostic work-up is advised. Magnetic resonance imaging of the spine is recommended. Potential causative pathogens should be identified by blood culture or biopsy before initiation of treatment with antibiotics. ${ }^{20}$ An extensive review of treatment options for spondylodiscitis is beyond the scope of this article.

\subsubsection{Acute vs chronic back pain}

Acute back pain is defined as back pain with a duration of less than 6 weeks. ${ }^{21}$ Although lumbalgia and ischialgia are common in the 
setting of strain or trauma, acute back pain can have inflammatory features resembling inflammatory back pain. However, unlike strain and trauma, sacroiliitis or spondylitis will usually not result in antalgic posture or limping. Likewise, neurologic symptoms are more indicative of degenerative disc disease, spinal canal stenosis or pain originating from facet joints. A referral strategy for IBD patients with acute back pain can be found in Figure S1. Overall, acute back pain is rarely caused by rheumatologic conditions, and patients should be referred to other musculoskeletal specialists.

Chronic back pain is generally defined as back pain with a duration of at least 6-12 weeks. ${ }^{21}$ Although back pain is often the main complaint in axial spondyloarthritis, only $5 \%$ of patients with chronic back pain have underlying axial spondyloarthritis. ${ }^{22,23}$ However, the prevalence of axial spondyloarthritis is higher in patients with chronic back pain and a closely related disease, such as IBD. ${ }^{12}$

\subsection{3 | Referral strategy for IBD patients with chronic back pain}

Based on an algorithm previously developed to assist in the early diagnosis of axial spondyloarthritis in all patients with chronic back pain, ${ }^{8}$ we designed an adapted referral algorithm for IBD patients.
Our decision tree was supported by calculations of post-test probabilities by applying formulas based on Bayes' theorem, as described in the original article. ${ }^{8}$

The previously developed algorithm utilises average representative sensitivities and specificities of several spondyloarthritis features, which were collected from the literature. As recognition of some of the spondyloarthritis features may be difficult for physicians who are less familiar with rheumatic disease, we simplified the algorithm to include inflammatory back pain, response to nonsteroidal anti-inflammatory drugs (NSAIDs) and human leucocyte antigen (HLA)-B27 status, which are important and well-known spondyloarthritis characteristics. C-reactive protein (CRP) elevation was not included because IBD may account for elevated CRP levels regardless of concomitant spondyloarthritis. As our tool will be used for screening, sensitivity was prioritised.

The cardinal symptom in axial spondyloarthritis is inflammatory back pain, which is present in most patients (Figure 1). ${ }^{24}$ Different sets of criteria used in clinical practice were proposed to identify patients with inflammatory back pain. Age at onset is an important differentiating factor in back pain. However, immunosuppressants, including anti-tumour necrosis factor (anti-TNF), may in some cases suppress inflammation and therefore postpone incipient

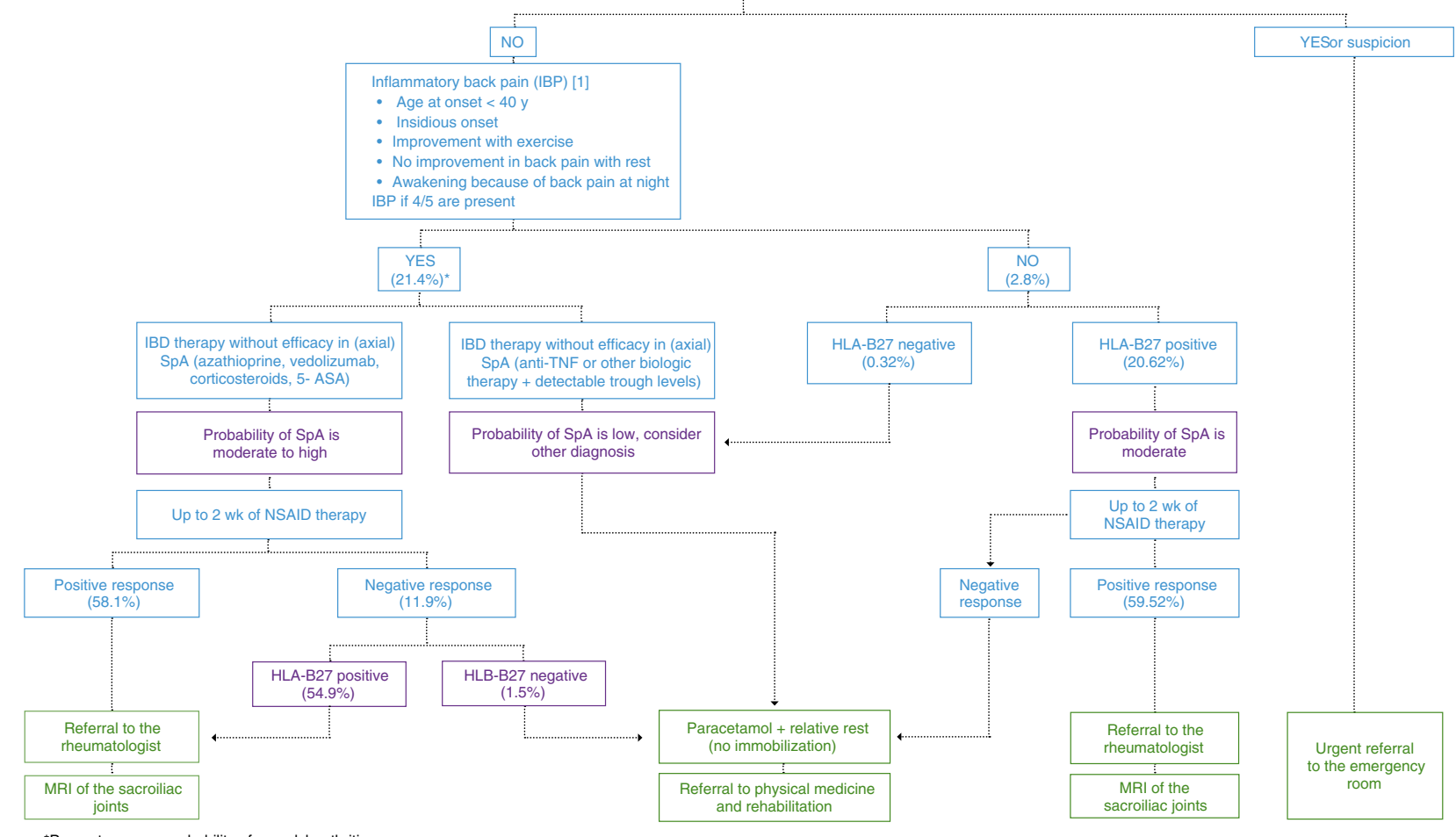

*Percentages are probability of spondyloarthritis

[1] Sieper J. et al Ann Rheum Dis. 2009.

FIGURE 1 Referral pattern for IBD patients with chronic back pain. Abbreviations: anti-TNF, anti-tumour necrosis factor; HLA, human leucocyte antigen; IBD, inflammatory bowel disease; IBP, inflammatory back pain; MRI, magnetic resonance imaging; NSAIDs, nonsteroidal anti-inflammatory drugs; SpA, spondyloarthritis. Percentages indicate post-test probabilities ${ }^{33}$ 
symptoms. ${ }^{25}$ To be recognised as having inflammatory back pain, patients had to present with four out of five of the following criteria: age at onset $<40$ years, insidious onset, improvement with exercise, no improvement in back pain with rest or awakening because of back pain at night. ${ }^{25}$

Axial spondyloarthritis prevalence in IBD patients was estimated at $8 \%$, which is the prevalence of symptomatic sacroiliitis in these patients. Asymptomatic sacroiliitis cannot be considered as axial spondyloarthritis and was therefore not included. When IBD patients who present with inflammatory back pain, receive anti-TNF therapy or other biologics with proven efficacy against axial spondyloarthritis and have detectable serum trough levels, onset of axial spondyloarthritis is improbable, although not impossible. In these patients, degenerative changes or fibromyalgia should be considered. Importantly, although ustekinumab showed efficacy in a small open-label study, larger randomised controlled trials have failed to validate these results. ${ }^{26-29}$ In patients receiving IBD treatments not considered as protective against spondyloarthritis (eg azathioprine, corticosteroids or vedolizumab), the probability of axial spondyloarthritis is moderate to high. ${ }^{30-32}$ In these patients, NSAID therapy should be considered. Although all NSAIDs are equally effective in treating axial spondyloarthritis, the optimal dose per product is necessary (Table 1). ${ }^{33}$ Even though IBD flares in patients using NSAIDs were reported in case reports and observational studies, randomised controlled trials and cohort studies have produced reassuring data regarding the short-term use of Cox-selective NSAIDs. ${ }^{34-36}$ Nevertheless, in patients with uncontrolled IBD, NSAIDs should be used cautiously.

Based on the estimated axial spondyloarthritis prevalence in IBD patients (pre-test probability) and the sensitivity and specificity of inflammatory back pain criteria, IBD patients with chronic inflammatory back pain have a probability of $21.4 \%$ of having axial

TAB LE 1 Comparable efficacy of each NSAID with $150 \mathrm{mg}$ of diclofenac, adapted from Dougados et $\mathrm{al}^{33}$

\begin{tabular}{|lr|}
\hline NSAID & Daily dose $(\mathbf{m g})$ \\
\hline Diclofenac & 150 \\
\hline Naproxen & 1000 \\
\hline Aceclofenac & 200 \\
\hline Celecoxib & 400 \\
\hline Etodolac & 600 \\
\hline Etoricoxib & 90 \\
\hline Flurbiprofen & 200 \\
\hline Ibuprofen & 2400 \\
\hline Indometacin & 150 \\
\hline Ketoprofen & 200 \\
\hline Meloxicam & 15 \\
\hline Nimesulide & 200 \\
\hline Phenylbutazone & 400 \\
\hline Piroxicam & 20 \\
\hline Tenoxicam & 20 \\
\hline
\end{tabular}

spondyloarthritis. Good response to NSAIDs results in a rise in the axial spondyloarthritis probability to $58.1 \%$. Lack of response to NSAIDs results in a decrease in the axial spondyloarthritis probability to $11.9 \%$; in these patients, determining the HLA-B27 status can provide an alternative route if suspicion remains high, with positive tests resulting in the axial spondyloarthritis probability of $54.9 \%$. Indeed, in patients with chronic low back pain, both HLA-B27 status and inflammatory back pain have proven to be good referral criteria. ${ }^{37}$ If inflammatory back pain is not present, the axial spondyloarthritis probability decreases to $2.8 \%$. Even if patients without inflammatory back pain are positive for HLA-B27, this probability only rises to $20.6 \%$. If these patients also have a good response to NSAIDs, a reasonable probability of $59.5 \%$ can be reached.

The sensitivities and specificities used in this paper are based on a high number of estimates from several large studies in IBD. Nevertheless, applying different sensitivities and specificities may lead to different probabilities. The post-test probabilities should be regarded as estimates and the validity of these algorithms should be tested in real-life setting. Additionally, convergence was limited by the inclusion of independent variables in the algorithm.

The referral algorithm presented here can be used to evaluate IBD patients presenting with chronic back pain and to identify which patients should be referred to specialists based on basic clinical criteria and one objective laboratory test. Because of the need of specific expertise in interpretation of imaging, the opinion of the committee was that imaging is not necessary before referral. Indeed, $\mathrm{X}$-rays of sacroiliac joints may give false-negative results in early disease stages and pelvic X-ray interpretation has proven unreliable and reader-dependent. ${ }^{38}$ Additionally, magnetic resonance imaging is expensive, in high demand and only reliably interpretable in patients with high suspicion of spondyloarthritis. Different types of mechanical stress, including physiological changes induced by pregnancy, may mimic sacroiliitis. These imaging techniques should only be requested and interpreted by experienced readers with full knowledge of the clinical background.

In conclusion, chronic back pain is a prevalent and multi-layered complaint which may be influenced by therapy. This algorithm provides a clinical tool to differentiate whether referral is needed by the use of pain pattern, the patient's IBD therapy, response to NSAID and the HLA-B27 status. Imaging is not essential in the initial work-up of any patient, as imaging quality and expertise may be required.

\section{2 | Peripheral arthritis}

Peripheral arthritis prevalence in IBD patients has been estimated at $13 \%$, being highest (25\%) in 20-30-year-old patients. ${ }^{12}$ Arthritis is characterised by swelling and pain, but redness and warmth are also frequent. Before diagnostic investigation, it is critical to collect the patient's medical history-including concomitant symptoms and previous joint flares-and to perform a complete clinical examination with attention to age, skin and distribution of affected joints. ${ }^{39}$ Symptoms of preceding enteric or genitourinary infection 
with pathogens such as Salmonella, Shigella, Yersinia, Campylobacter, Clostridium or Chlamydia trachomatis should be inquired for.

Typically, peripheral spondyloarthritis has an oligoarticular distribution with a preferential localisation in large joints of lower limbs in young patients. ${ }^{40}$ In contrast, rheumatoid arthritis (RA) and (drug-induced) systemic lupus erythematosus (SLE) display a more symmetrical distribution, generally localised on small joints of hands and feet. $^{41}$

\subsection{1 | Monoarthritis in IBD patients}

Monoarthritis of a joint is a common clinical presentation in any population. However, the differential diagnosis is broad, and about half of patients with acute monoarthritis do not have a definitive diagnosis within 2 years. ${ }^{42}$

In the case of monoarthritis in IBD patients, exclusion of trauma and/or septic arthritis is essential. The latter can lead to joint destruction and sepsis with multiple organ failure, especially in patients treated with immunosuppressants or having other risk factors (eg, older age, diabetes mellitus, surgery or prosthetic joint). ${ }^{43}$ Patients with clinical indications of possible septic arthritis, such as fever, skin infection or entry wound, should be referred to the emergency room for blood analysis, evacuation of purulent material, puncture and blood culture before initiation of antibiotics. A white blood cell (WBC) count of $>50000$ cells $/ \mu$ l with $\geq 90 \%$ neutrophils in the puncture fluid may be indicative of septic arthritis. ${ }^{43}$ Moreover, the sensitivity of a positive synovial culture is 75\%-95\% for septic arthritis. ${ }^{43}$ Other blood analyses are commonly performed for diagnosis purposes (WBC count, CRP, sedimentation, uric acid, rheumatoid factor [RF] and anti-cyclic citrullined peptide [anti-CCP] levels).

\section{Large joint monoarthritis}

Since there are no established referral strategies for peripheral arthritis, the development of the decision tree for IBD patients with large joint monoarthritis was based on expert opinion, literature and experience (Figure 2).

Patients with large joint monoarthritis (knee, ankle, hip, shoulder, elbow or wrist) should be referred to a rheumatologist for articular puncture with synovial fluid evacuation (Figure 2). The purpose of this procedure is both therapeutic and diagnostic (cell count, culture and/or characterisation of crystals). Treatments, such as oral corticosteroids, disease-modifying anti-rheumatic drugs and antibiotics, should not be initiated before the puncture, as they may mask distribution, systemic inflammation and in some cases auto-immune serology, thereby complicating prompt diagnosis.

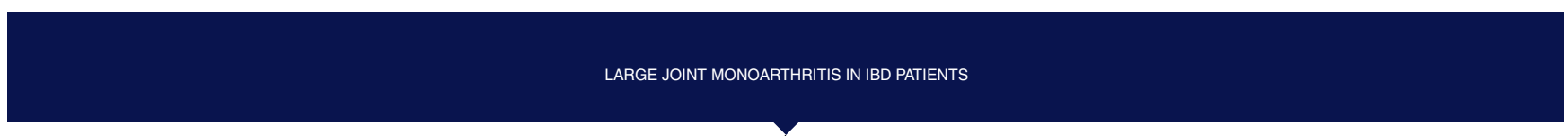

Septic arthritis?

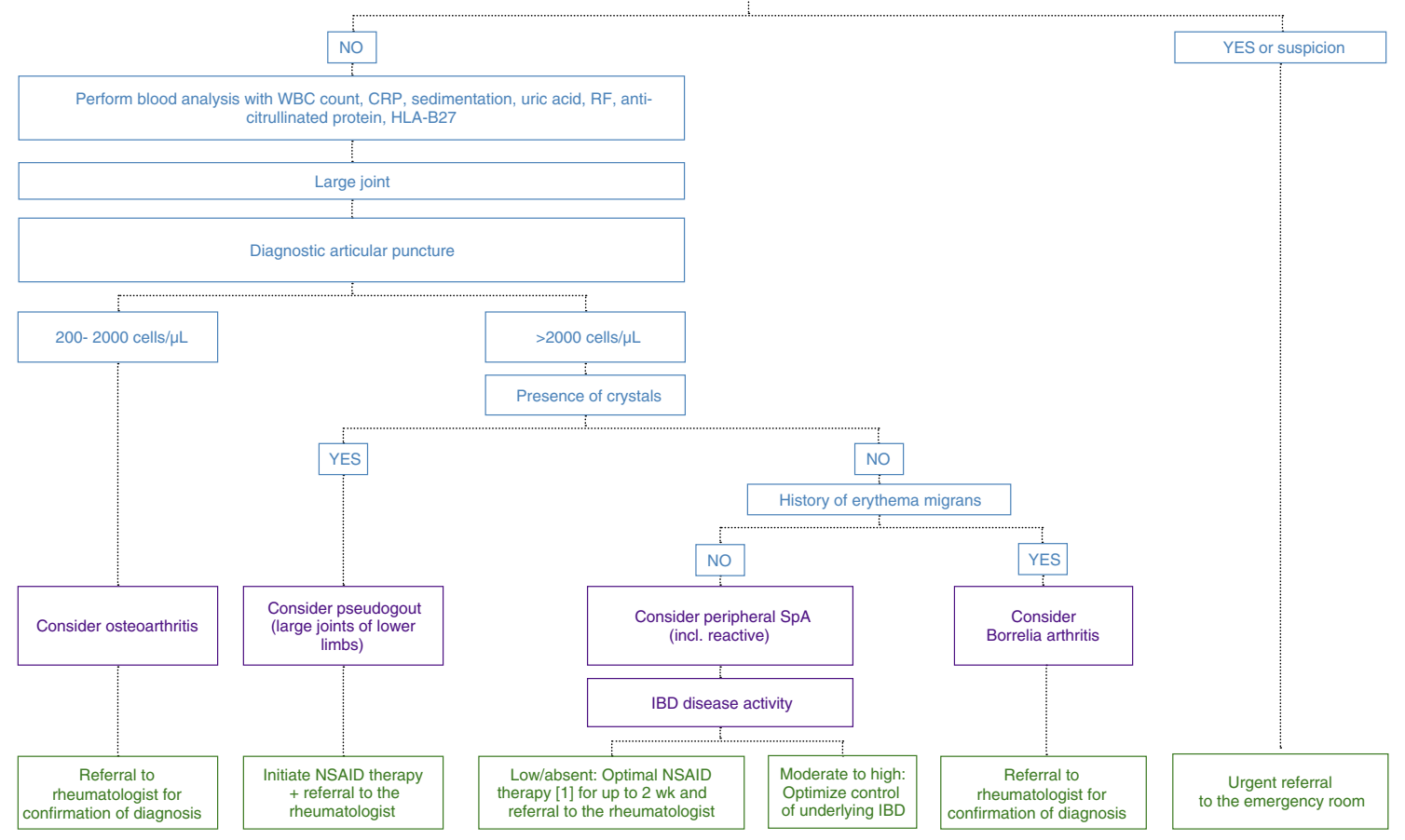

[1]Dougados et al. Ann Rheum Dis. 2011.

FIG URE 2 Referral pattern for IBD patients with large joint monoarthritis. Abbrevations: CRP, C-reactive protein; IBD, inflammatory bowel disease; NSAIDs, nonsteroidal anti-inflammatory drugs; RF, rheumatoid factor; SpA, spondyloarthritis; WBC, white blood cell ${ }^{33}$ 


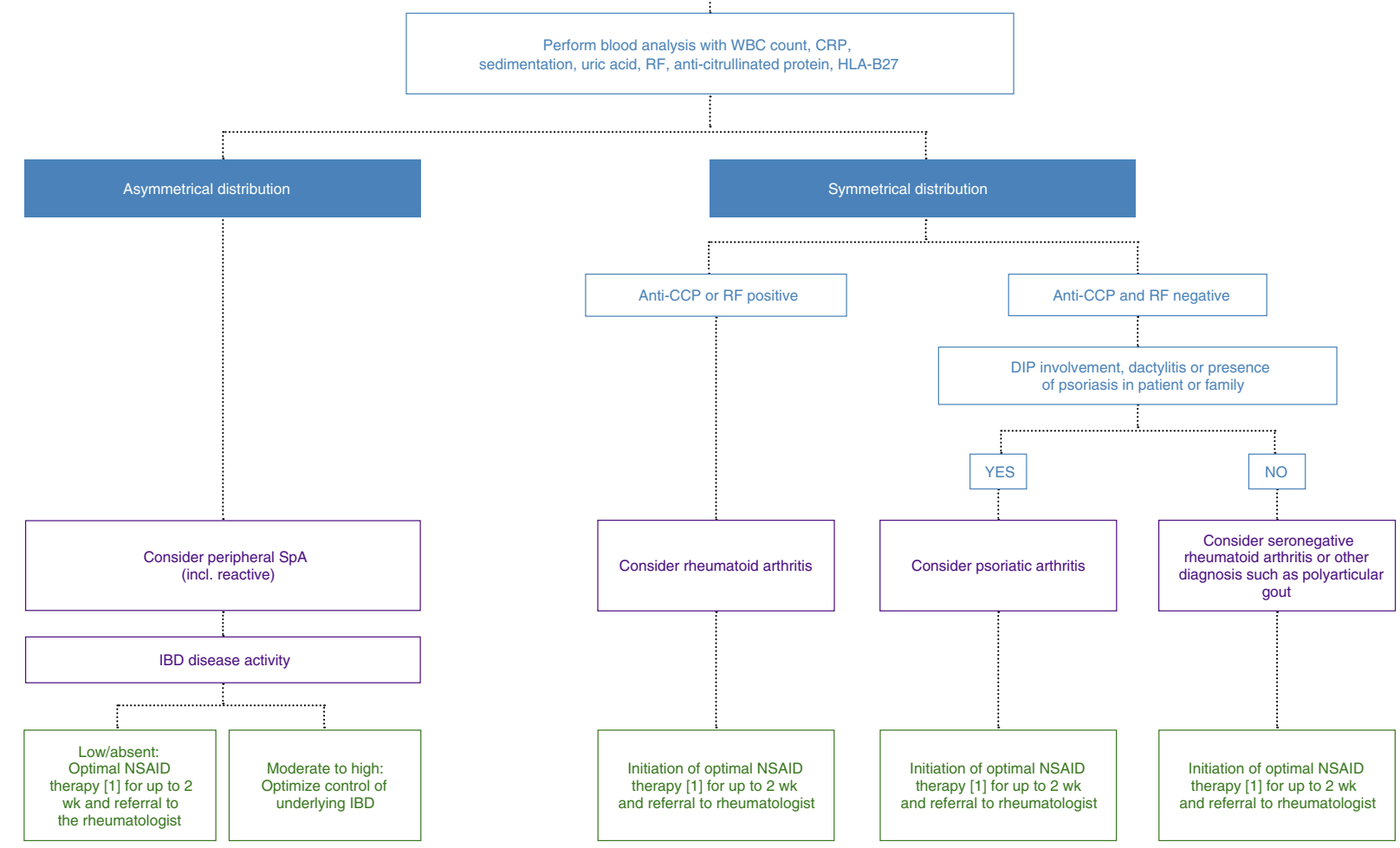

[1] Dougados et al. Ann Rheum Dis. 2011.

FIGURE 3 Referral pattern for IBD patients with oligo- or polyarticular arthritis. Abbreviations: CCP, cyclic citrullined peptide; CRP, C-reactive protein; DIP, distal interphalangeal; IBD, inflammatory bowel disease; NSAIDs, nonsteroidal anti-inflammatory drugs; RA, rheumatoid arthritis; RF, rheumatoid factor; SpA, spondyloarthritis; WBC, white blood cell ${ }^{33}$

Synovial fluid containing $>2000 \mathrm{WBC} / \mu \mathrm{l}$ and $>75 \%$ polymorphonuclear cells is indicative of inflammatory joint disease, whereas synovial fluid containing $<2000 \mathrm{WBC} / \mu \mathrm{l}$ is usually indicative of mechanical joint disease/osteoarthritis. ${ }^{44,45}$ In case of inflammatory joint disease, polarisation microscopy should be used to detect the presence of monosodium urate or calcium pyrophosphate dihydrate crystals, indicative of gout and pseudogout, respectively. ${ }^{46}$ If crystals are present, (pseudo)-gout should be considered, local corticosteroid infiltration administered, and NSAID or colchicine therapy initiated. Referral to a rheumatologist is recommended. If no crystals are detected, $B$ burgdorferi arthritis should be suspected in patients with current or previous erythema migrans or documented tick bites as a late manifestation of Lyme disease. $B$ burgdorferi serology should not be done in every patient as the screening assay has high sensitivity at the expense of specificity. ${ }^{47}$ The suspicion of $B$ burgdorferi will be influenced by the local incidence rate of Lyme's disease. When in doubt, the presence of DNA in the synovial fluid can be tested using polymerase chain reaction. In the absence of erythema migrans history, peripheral spondyloarthritis should be considered and optimal NSAID therapy or low-dose corticosteroids should be initiated. Referral to a rheumatologist is recommended. An extensive review of treatment of peripheral spondyloarthritis is beyond the scope of this article. Nevertheless, methotrexate, leflunomide and sulphasalazine are slow-acting agents, which are useless for immediate symptom control but may have an evaluable clinical benefit only after 8-12 weeks.

\section{Small joint monoarthritis}

The approach in case of small joint monoarthritis is more difficult, as diagnostic puncture is rarely an option. The differential diagnosis is mainly based on serology (Figure S2). In RF- or anti-CCP-positive patients, early RA diagnosis is possible, although the most frequent initial presentation of RA is symmetrical polyarthritis. ${ }^{48}$ In RF- and anti-CCP-negative patients, osteoarthritis, peripheral spondyloarthritis or gout are potential diagnoses. Nevertheless, a small portion of patients exhibit seronegative RA.

In case of single arthritis at the level of the hands in combination with bony changes of other proximal interphalangeal and distal interphalangeal joints, erosive hand osteoarthritis should be considered in middle-aged or elderly patients. ${ }^{49}$ The presence of tophi or the typical localisation at the first toe metatarsophalangeal joint can advocate in favour of gout, even in the absence of high serum uric acid in the acute phase. Importantly, monoarthritis 
in IBD patients can be the first symptom of systemic disease and subsequently spread towards other joints or the axial skeleton.

\subsubsection{Oligo- or polyarthritis in IBD patients}

When several joints are involved, both distribution and auto-immune serology are crucial. If articular puncture of a large joint is possible, the latter should be performed to refine diagnosis (Figure 3).

\subsection{3 | Arthralgia in IBD patients}

Arthralgia is the presence of one or more painful joints without objective signs of inflammation, such as swelling, redness or warmth. An inflammatory cause should be suspected if pain is worst during the night and in the morning, the patient has morning stiffness and feels better with exercise. A mechanical cause should be suspected if pain is worsening throughout the day, the patient has pain upon weight bearing and feels better with rest. In case of mechanical pain, a "wait and see" approach with paracetamol for pain relief is recommended (Figure 4 and Figure S3).

\section{Monoarticular arthralgia}

In patients with persistent monoarticular arthralgia caused by mechanical pain and in patients with inflammatory arthralgia, the presence of inflammatory activity should be assessed using ultrasound. In the absence of systemic inflammation, arthralgia related to overuse or osteoarthritis should be considered. In the case of positive ultrasound results, monoarthritis should be considered, and treatment with NSAIDs should be initiated. In both cases, referral to a rheumatologist is recommended (Figure S3).

\section{Oligoarticular and polyarticular arthralgia}

In patients with persistent oligoarticular and polyarticular arthralgia caused by mechanical pain, osteoarthritis and fibromyalgia should be considered (Figure 3).

In patients with inflammatory oligoarticular and polyarticular arthralgia, erythrocyte sedimentation rates should be evaluated (Figure 3). In patients with normal sedimentation, arthralgia is often related to treatment or discontinuation of therapy in absence of systemic inflammation. Arthralgia and/or myalgia have been reported in $14 \%$ of azathioprine-treated patients, of whom
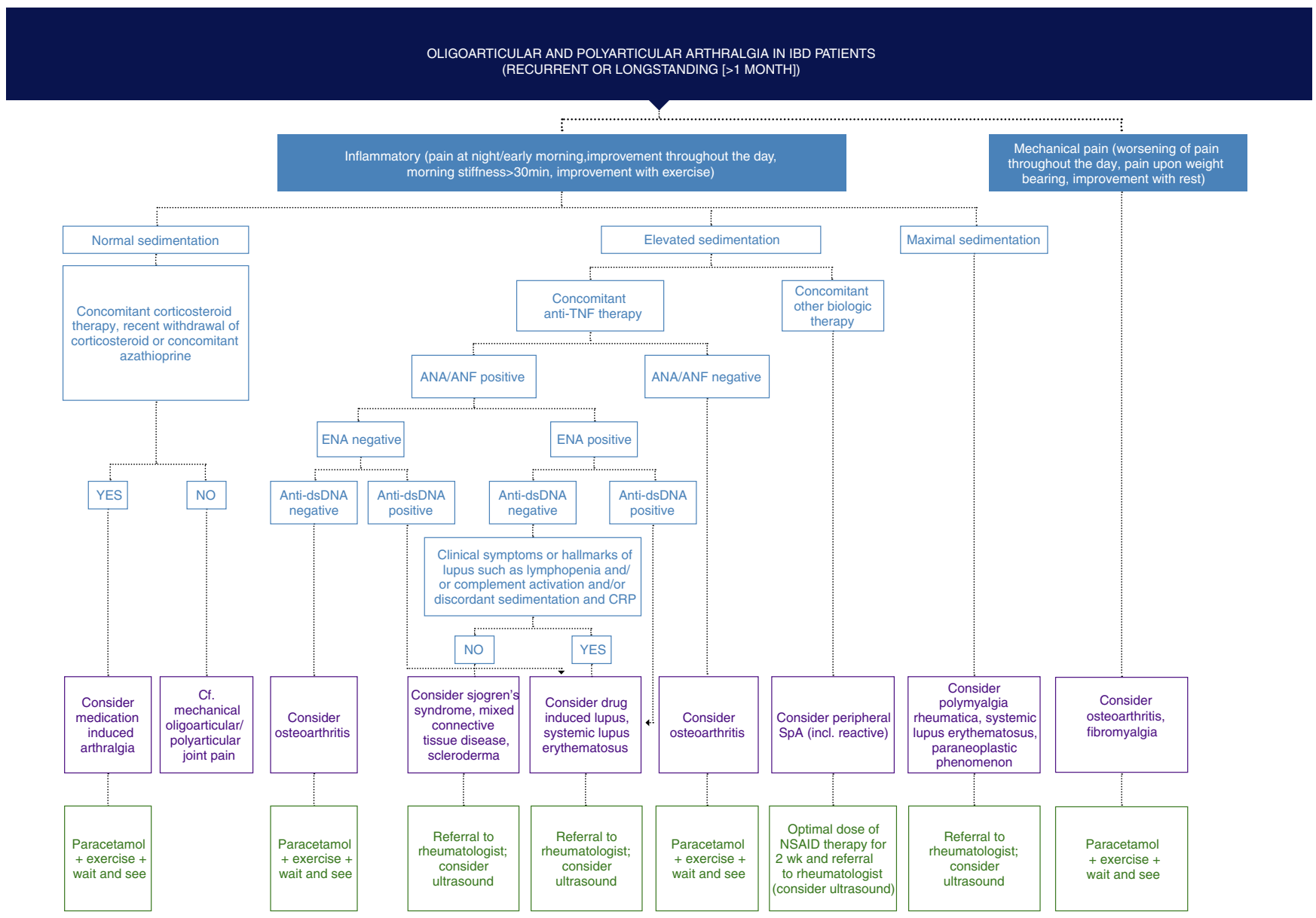

FIG URE 4 Referral pattern for IBD patients with oligoarticular and polyarticular arthralgia. Abbreviations: ANA, antinuclear antibody; ANF, antinuclear factor; anti-dsDNA, anti-double-stranded DNA; Cf., confer; CRP, C-reactive protein; DD, differential diagnosis; ENA, extractable nuclear antigens; IBD, inflammatory bowel disease; NSAIDs, nonsteroidal anti-inflammatory drugs; SpA, spondyloarthritis; TNF, tumour necrosis factor; WBC, white blood cell 
$68 \%$ tolerated a switch to mercaptopurine. ${ }^{50}$ Likewise, the introduction of infliximab entails a risk of transitory arthralgia, ${ }^{51}$ and the withdrawal of corticosteroids may involve a temporary increase of musculoskeletal symptoms. ${ }^{52}$ In patients with elevated sedimentation, possible culprits may be sulphasalazine or anti-TNF therapies. ${ }^{53,54}$ Although immunogenicity has been described with all anti-TNFs, infliximab seems to be the most immunogenic. ${ }^{55,56}$ Importantly, anti-TNF therapy primarily induces immunoglobulin (Ig) M, rather than IgG antibodies. Therefore, isolated antinuclear factor (ANF) elevation is of limited significance in absence of clinical symptoms. Moreover, while ANF induction is common, druginduced SLE is rare. ${ }^{55}$ Drug-induced SLE should be considered in patients with arthralgia who were treated with anti-TNF and present with anti-double-stranded deoxyribonucleic acid (anti-dsDNA) IgGs, hypocomplementaemia, lymphopenia, discrepant sedimentation and CRP or clinical signs of SLE. However, even in patients receiving anti-TNF therapies, idiopathic SLE might have been present before drug initiation. Although clinical symptoms may disappear rapidly after discontinuation of the culprit drug, laboratory abnormalities may remain for some time. In patients with elevated sedimentation receiving other biologics, the response to NSAIDs or low-dose corticosteroids should be evaluated prior to referral to a rheumatologist. The rheumatologist may consider ultrasound to assess the presence of inflammatory activity. In patients exhibiting very high sedimentation, an infectious locus should be considered. However, in the presence of concomitant proximal muscle weakness, prompt referral to a rheumatologist is recommended because these symptoms may be indicative of polymyalgia rheumatica or other systemic auto-immune disease.

\section{4 | CONCLUSION}

In this paper, we presented straightforward referral algorithms for IBD patients with spondyloarthritis symptoms, developed by a panel of Belgian gastroenterologists and rheumatologists. The main focus was on IBD patients with chronic back pain, large joint monoarthritis and arthralgia. The proposed strategies allow a clear evaluation for referral based on basic clinical criteria in combination with laboratory tests to identify which patients should be referred to emergency room, physical medicine or rheumatologist. The proposed strategies are specific enough to evaluate IBD patients with joint symptoms without overloading rheumatologists with unnecessary referrals.

A limitation of this study is the use of different terminologies in gastroenterology and rheumatology regarding axial spondyloarthritis, leading to a potential underestimation of the axial spondyloarthritis prevalence. Moreover, we developed referral strategies based on expert opinion because the absence of scientific literature regarding the referral of IBD patients with peripheral arthritis is striking. Overall, the proposed algorithms may help gastroenterologists to make a broad clinical differential diagnosis and to adequately select patients for referral.

\section{ACKNOWLEDGEMENTS}

Claire Verbelen of XPE Pharma \& Science, Belgium, provided medical writing support.

Declaration of personal interests: GV received a research grant from Ghent University. GV received grants or speakers fees from Pfizer and AbbVie. DE and RL are supported by grants from the Flanders Research Foundation (FWO-Vlaanderen). DE is supported by the Research Council of Ghent University. DE is also a member of a multidisciplinary research platform (MRP) of Ghent University. DE and $\mathrm{RL}$ are supported by Interuniversity Attraction Pole (IUAP) grant Devrepair from the Belspo Agency (project P7/07). DE received grants or speakers fees from Boehringer Ingelheim, Pfizer, UCB, Merck, Novartis, Janssen and AbbVie. FVDB received consultancy and/or speaker fees from AbbVie, Celgene, Janssen, Merck, Novartis, Pfizer and UCB. Leuven Research and Development, the technology transfer office of KU Leuven has received speaker's and consultancy fees on behalf of RL from AbbVie, Boehringer-Ingelheim, Celgene, Janssen, Novartis, Merck, Pfizer and UCB and research grants from BoehringerIngelheim, Celgene and Pfizer. EL received fees for Research Grant: Takeda, Pfizer; Educational Grant: AbbVie, MSD, Takeda; Speaker Fees: Abbott, AbbVie, AstraZeneca, Ferring, MSD, Chiesi, Falk, Takeda, Hospira, Janssen, Pfizer; Advisory Board: Abbott, AbbVie, Ferring, MSD, Mitsubishi Pharma, Takeda, Celltrion, Celgene, Hospira, Janssen; Consultant: AbbVie, Elli-Lily. MDV received educational grants from Abbvie and Ferring and a consultancy fee from Abbvie, Takeda and Janssen. SV has served as a speaker for AbbVie, MSD, Pfizer, Takeda, Hospira, Janssen and Ferring; received consultant fees from AbbVie, MSD, Pfizer, Takeda, Hospira, Ferring, Mundipharma, Second Genome, Galapagos, Genentech/Roche, Janssen and Celgene; served as an advisory board member for AbbVie, MSD, Pfizer, Takeda, Hospira, Ferring, Mundipharma, Second Genome, Galapagos, Genentech/Roche, Janssen and Celgene; and received research grants from MSD, AbbVie, Pfizer and Takeda. CR has received speaker's fees or consultancy fees from Abbvie, BMS, MSD, Pfizer and UCB.

\section{AUTHORSHIP}

Guarantor of the article: Gaëlle Varkas, Dirk Elewaut and Martine De Vos.

Author contributions: GV, CR, EL, FVDB, RL, SV, DE, MDV were involved in the study concept and design (Delphi exercise). GV, FVDB, DE, MDV acquired the data. GV, CR, EL, FVDB, RL, SV, DE, MDV analysed and interpreted the data. GV, DE, MDV collaborated with Claire Verbelen and prepared the manuscript. GV, CR, EL, FVDB, RL, $\mathrm{SV}, \mathrm{DE}, \mathrm{MDV}$ revised the manuscript. All authors approved the final version of the manuscript.

\section{ORCID}

Gaëlle Varkas (iD https://orcid.org/0000-0001-7606-9256

Martine De Vos iD https://orcid.org/0000-0003-3598-4153 


\section{REFERENCES}

1. Levine JS, Burakoff R. Extraintestinal manifestations of inflammatory bowel disease. Gastroenterol Hepatol (N Y). 2011;7:235-241.

2. Harbord M, Annese V, Vavricka SR, et al. The first European evidence-based consensus on extra-intestinal manifestations in inflammatory bowel disease. J Crohns Colitis. 2016;10:239-254.

3. Parma A, Cometi L, Leone MC, et al. One year in review 2016: spondyloarthritis. Clin Exp Rheumatol. 2017;35:3-17.

4. Faustini F, Zoli A, Ferraccioli GF. Immunologic and genetic links between spondylarthropathies and inflammatory bowel diseases. Eur Rev Med Pharmacol Sci. 2009;13(Suppl 1):1-9.

5. Rudwaleit M, Baeten D. Ankylosing spondylitis and bowel disease. Best Pract Res Clin Rheumatol. 2006;20:451-471.

6. van Sommeren S, Janse M, Karjalainen J, et al. Extraintestinal manifestations and complications in inflammatory bowel disease: from shared genetics to shared biological pathways. Inflamm Bowel Dis. 2014;20:987-994.

7. Sieper J, Rudwaleit M, Baraliakos X, et al. The assessment of spondyloarthritis international society (asas) handbook: a guide to assess spondyloarthritis. Ann Rheum Dis. 2009;68:ii1-ii44.

8. Rudwaleit M, van der Heijde D, Khan MA, Braun J, Sieper J. How to diagnose axial spondyloarthritis early. Ann Rheum Dis. 2004;63:535-543.

9. Braun J, Sieper J, Breban M, et al. Anti-tumour necrosis factor alpha therapy for ankylosing spondylitis: International experience. Ann Rheum Dis. 2002;61:iii51-60.

10. Poddubnyy D, Haibel H, Listing J, et al. Baseline radiographic damage, elevated acute-phase reactant levels, and cigarette smoking status predict spinal radiographic progression in early axial spondylarthritis. Arthritis Rheum. 2012;64:1388-1398.

11. Dougados M, Demattei C, van den Berg R, et al. Rate and predisposing factors for sacroiliac joint radiographic progression after a two-year follow-up period in recent-onset spondyloarthritis. Arthritis Rheumatol. 2016;68:1904-1913.

12. Karreman MC, Luime JJ, Hazes J, Weel A. The prevalence and incidence of axial and peripheral spondyloarthritis in inflammatory bowel disease: a systematic review and meta-analysis. J Crohns Colitis. 2017:11:631-642.

13. De Vos M, Mielants H, Cuvelier C, Elewaut A, Veys E. Long-term evolution of gut inflammation in patients with spondyloarthropathy. Gastroenterology. 1996;110:1696-1703.

14. Mielants H, Veys EM, Cuvelier C, et al. The evolution of spondyloarthropathies in relation to gut histology. li. Histological aspects. J Rheumatol. 1995;22:2273-2278.

15. Lanna C, Ferrari MdLA, Rocha SL, Nascimento E, de Carvalho M, da Cunha AS. A cross-sectional study of 130 Brazilian patients with crohn's disease and ulcerative colitis: analysis of articular and ophthalmologic manifestations. Clin Rheumatol. 2008;27:503-509.

16. McEniff N, Eustace S, McCarthy C, O'Malley M, O'Morain CA, Hamilton S. Asymptomatic sacroiliitis in inflammatory bowel disease assessment by computed tomography. Clin Imaging. 1995;19:258-262.

17. Steer $\mathrm{S}$, Jones $\mathrm{H}$, Hibbert J, et al. Low back pain, sacroiliitis, and the relationship with HLA-b27 in Crohn's disease. J Rheumatol. 2003;30:518-522.

18. Leclerc-Jacob S, Lux G, Rat AC, et al. The prevalence of inflammatory sacroiliitis assessed on magnetic resonance imaging of inflammatory bowel disease: a retrospective study performed on 186 patients. Aliment Pharmacol Ther. 2014;39:957-962.

19. Gotler J, Amitai MM, Lidar M, Aharoni D, Flusser G, Eshed I. Utilizing mr enterography for detection of sacroiliitis in patients with inflammatory bowel disease. J Magn Reson Imaging. 2015;42:121-127.

20. Berbari EF, Kanj SS, Kowalski TJ, et al. 2015 infectious diseases society of America (idsa) clinical practice guidelines for the diagnosis and treatment of native vertebral osteomyelitis in adults. Clin Infect Dis. 2015;61:e26-46.

21. Deyo RA, Weinstein JN. Low back pain. N Engl J Med. 2001;344:363-370.

22. O'Shea FD, Boyle E, Salonen DC, et al. Inflammatory and degenerative sacroiliac joint disease in a primary back pain cohort. Arthritis Care Res (Hoboken). 2010;62:447-454.

23. Underwood MR, Dawes P. Inflammatory back pain in primary care. Br J Rheumatol. 1995;34:1074-1077.

24. Mau W, Zeidler H, Mau R, et al. Clinical features and prognosis of patients with possible ankylosing spondylitis. Results of a 10-year followup. J Rheumatol. 1988;15:1109-1114.

25. Sieper J, van der Heijde D, Landewe R, et al. New criteria for inflammatory back pain in patients with chronic back pain: a real patient exercise by experts from the Assessment of SpondyloArthritis international Society (ASAS). Ann Rheum Dis. 2009;68(6):784-788.

26. van der Heijde D, Dijkmans B, Geusens P, et al. Efficacy and safety of infliximab in patients with ankylosing spondylitis: results of a randomized, placebo-controlled trial (assert). Arthritis Rheum. 2005;52:582-591.

27. van der Heijde $D$, Kivitz A, Schiff $M H$, et al. Efficacy and safety of adalimumab in patients with ankylosing spondylitis: results of a multicenter, randomized, double-blind, placebo-controlled trial. Arthritis Rheum. 2006;54:2136-2146.

28. Poddubnyy D, Hermann KG, Callhoff J, Listing J, Sieper J. Ustekinumab for the treatment of patients with active ankylosing spondylitis: results of a 28-week, prospective, open-label, proof-ofconcept study (topas). Ann Rheum Dis. 2014;73:817-823.

29. Sandborn W, Gasink C, Blank M, et al. O-001a multicenter, doubleblind, placebo-controlled phase 3 study of ustekinumab, a human IL-12/23p40 mab, in moderate-service crohn's disease refractory to anti-tfnalpha: Uniti-1. Inflamm Bowel Dis. 2016;22(Suppl 1):S1.

30. Haibel H, Fendler C, Listing J, Callhoff J, Braun J, Sieper J. Efficacy of oral prednisolone in active ankylosing spondylitis: results of a double-blind, randomised, placebo-controlled short-term trial. Ann Rheum Dis. 2014;73:243-246.

31. Varkas G, Thevissen K, De Brabanter G, et al. An induction or flare of arthritis and/or sacroiliitis by vedolizumab in inflammatory bowel disease: a case series. Ann Rheum Dis. 2016;76:878-881.

32. Tadbiri S, Peyrin-Biroulet L, Serrero M, et al. Impact of vedolizumab therapy on extra-intestinal manifestations in patients with inflammatory bowel disease: a multicentre cohort study nested in the observ-ibd cohort. Aliment Pharmacol Ther. 2018;47:485-493.

33. Dougados M, Paternotte S, Braun J, et al. Asas recommendations for collecting, analysing and reporting nsaid intake in clinical trials/ epidemiological studies in axial spondyloarthritis. Ann Rheum Dis. 2011;70:249-251.

34. Bonner GF, Fakhri A, Vennamaneni SR. A long-term cohort study of nonsteroidal anti-inflammatory drug use and disease activity in outpatients with inflammatory bowel disease. Inflamm Bowel Dis. 2004;10:751-757.

35. Sandborn WJ, Stenson WF, Brynskov J, et al. Safety of celecoxib in patients with ulcerative colitis in remission: a randomized, placebocontrolled, pilot study. Clin Gastroenterol Hepatol. 2006;4:203-211.

36. El Miedany Y, Youssef S, Ahmed I, El Gaafary M. The gastrointestinal safety and effect on disease activity of etoricoxib, a selective cox-2 inhibitor in inflammatory bowel diseases. Am J Gastroenterol. 2006;101:311-317.

37. Sieper J, Rudwaleit M. Early referral recommendations for ankylosing spondylitis (including pre-radiographic and radiographic forms) in primary care. Ann Rheum Dis. 2005;64:659-663.

38. van Tubergen A, Heuft-Dorenbosch L, Schulpen G, et al. Radiographic assessment of sacroiliitis by radiologists and rheumatologists: does training improve quality? Ann Rheum Dis. 2003;62:519-525 
39. Desforges JF, Baker DG, Schumacher HR. Acute monoarthritis. N Engl J Med. 1993;329:1013-1020.

40. Gladman DD. Clinical aspects of the spondyloarthropathies. Am J Med Sci. 1998;316:234-238.

41. Cervera R, Khamashta MA, Hughes GR. The euro-lupus project: Epidemiology of systemic lupus erythematosus in Europe. Lupus. 2009;18:869-874.

42. Jeong H, Kim AY, Yoon HJ, et al. Clinical courses and predictors of outcomes in patients with monoarthritis: a retrospective study of 171 cases. Int J Rheum Dis. 2014;17:502-510.

43. Margaretten ME, Kohlwes J, Moore D, Bent S. Does this adult patient have septic arthritis? JAMA. 2007;297:1478-1488.

44. Shmerling RH, Delbanco TL, Tosteson AN, Trentham DE. Synovial fluid tests. What should be ordered? JAMA. 1990;264:1009-1014.

45. Altman R, Asch E, Bloch D, et al. Development of criteria for the classification and reporting of osteoarthritis. Classification of osteoarthritis of the knee. Diagnostic and therapeutic criteria committee of the American rheumatism association. Arthritis Rheum. 1986;29:1039-1049.

46. Zhang W, Doherty M, Pascual E, et al. Eular evidence based recommendations for gout. Part i: diagnosis. Report of a task force of the standing committee for international clinical studies including therapeutics (escisit). Ann Rheum Dis. 2006;65:1301-1311.

47. Schenk J, Doebis C, Küsters U, von Baehr V. Evaluation of a new multiparametric microspot array for serodiagnosis of lyme borreliosis. Clin Lab. 2015;61:1715-1725.

48. Schellekens GA, Visser H, De Jong B, et al. The diagnostic properties of rheumatoid arthritis antibodies recognizing a cyclic citrullinated peptide. Arthritis Rheum. 2000;43:155-163.

49. Verbruggen G, Wittoek R, Vander Cruyssen B, Elewaut D. Morbid anatomy of 'erosive osteoarthritis' of the interphalangeal finger joints: an optimised scoring system to monitor disease progression in affected joints. Ann Rheum Dis. 2010;69:862-867.

50. Hindorf $U$, Johansson $M$, Eriksson A, Kvifors $E$, Almer SH. Mercaptopurine treatment should be considered in azathioprine intolerant patients with inflammatory bowel disease. Aliment Pharmacol Ther. 2009;29:654-661.
51. Lichtenstein L, Ron Y, Kivity S, et al. Infliximab-related infusion reactions: systematic review. J Crohns Colitis. 2015;9:806-815.

52. Rutgeerts PJ. Review article: the limitations of corticosteroid therapy in Crohn's disease. Aliment Pharmacol Ther. 2001;15:1515-1525.

53. Gunnarsson I, Kanerud L, Pettersson E, Lundberg I, Lindblad S, Ringertz B. Predisposing factors in sulphasalazine-induced systemic lupus erythematosus. Br J Rheumatol. 1997;36:1089-1094.

54. Ramos-Casals M, Brito-Zerón P, Muñoz S, et al. Autoimmune diseases induced by TNF-targeted therapies: analysis of 233 cases. Medicine (Baltimore). 2007;86:242-251.

55. De Rycke L, Baeten D, Kruithof E, Van den Bosch F, Veys EM, De Keyser F. The effect of TNFalpha blockade on the antinuclear antibody profile in patients with chronic arthritis: biological and clinical implications. Lupus. 2005;14:931-937.

56. De Bandt M, Sibilia J, Le Loët X, et al. Systemic lupus erythematosus induced by anti-tumour necrosis factor alpha therapy: a French national survey. Arthritis Res Ther. 2005;7:R545-R551.

\section{SUPPORTING INFORMATION}

Additional supporting information will be found online in the Supporting Information section at the end of the article.

How to cite this article: Varkas G, Ribbens C, Louis E, et al. Expert consensus: practical algorithms for management of inflammatory bowel disease patients presenting with back pain or peripheral arthropathies. Aliment Pharmacol Ther. 2019;50:1204-1213. https://doi.org/10.1111/apt.15519 